\title{
Circular stapling anastomosis with indocyanine green fluorescence imaging for cervical esophagogastric anastomosis after thoracoscopic esophagectomy: A propensity score-matched analysis
}

Yuji Shishido ( $\square$ y-shishido@tottori-u.ac.jp )

Tottori University Faculty of Medicine

Tomoyuki Matsunaga

Tottori University Faculty of Medicine

Masahiro Makinoya

Tottori University Faculty of Medicine

Wataru Miyauchi

Tottori University Faculty of Medicine

Shota Shimizu

Tottori University Faculty of Medicine

Kozo Miyatani

Tottori University Faculty of Medicine

Chihiro Uejima

Tottori University Faculty of Medicine

Masaki Morimoto

Tottori University Faculty of Medicine

Yuki Murakami

Tottori University Faculty of Medicine

Takehiko Hanaki

Tottori University Faculty of Medicine

Kyoichi Kihara

Tottori University Faculty of Medicine

Manabu Yamamoto

Tottori University Faculty of Medicine

Naruo Tokuyasu

Tottori University Faculty of Medicine

Shuichi Takano

Tottori University Faculty of Medicine 


\section{Teruhisa Sakamoto}

Tottori University Faculty of Medicine

Hiroaki Saito

Japanese Red Cross Tottori Hospital

\section{Toshimichi Hasegawa}

Tottori University Faculty of Medicine

\section{Yoshiyuki Fujiwara}

Tottori University Faculty of Medicine

\section{Research Article}

Keywords: esophageal cancer, thoracoscopic esophagectomy, cervical esophagogastric anastomosis, indocyanine green fluorescence imaging, circular stapling anastomosis, anastomotic leakage, anastomotic stenosis, propensity score matching

Posted Date: January 27th, 2022

DOI: https://doi.org/10.21203/rs.3.rs-1262279/v1

License: (c) (1) This work is licensed under a Creative Commons Attribution 4.0 International License. Read Full License 


\section{Abstract}

Background: Thoracoscopic esophagectomy has been widely used worldwide as a curative surgery for esophageal cancer, but complications, such as anastomotic leakage and stenosis remain a major concern. Therefore, this study aimed to evaluate the efficacy of circular stapling anastomosis with indocyanine green (ICG) fluorescence imaging, which was standardized for cervical esophagogastric anastomosis after thoracoscopic esophagectomy.

Methods: In total, 121 patients with esophageal cancer who underwent thoracoscopic esophagectomy with radical lymph node dissection and cervical esophagogastric anastomosis between November 2009 and December 2020 at Tottori University Hospital were enrolled in this study. Patients who underwent surgery before the anastomotic method was standardized were included in the classical group $(n=82)$, and patients who underwent surgery after standardization were included in the ICG circular group ( $\mathrm{n}=$ 39). We compared the short-term postoperative outcomes, including anastomotic complications, between the two groups using propensity matched analysis, and evaluated the risk factors for anastomotic leakage using logistic regression analyses.

Results: Of 121 patients, 33 were respectively included in each group after propensity score matching. The clinicopathological characteristics of patients between the two groups did not differ after matching. In perioperative outcomes, a significantly higher proportion of patients who underwent surgery using the laparoscopic approach $(P<0.001)$ and narrow gastric tube $(P=0.003)$, as well as those who had a lower volume of blood loss $(P=0.009)$ in the ICG circular group were seen after matching. Moreover, the ICG circular group had a significantly lower incidence of anastomotic leakage (39\% vs. $9 \%, P=0.004)$ and anastomotic stenosis ( $46 \%$ vs. $21 \%, P=0.037$ ) and a shorter postoperative hospital stay (30 vs. 20 days, $P<0.001)$ than the classical group. In the multivariate analysis, anastomotic method was an independent risk factor for anastomotic leakage after thoracoscopic esophagectomy $(P=0.013)$.

Conclusions: Circular stapling anastomosis with ICG fluorescence imaging is effective in reducing complications including anastomotic leakage and stenosis.

\section{Background}

Esophageal cancer is the ninth most commonly diagnosed cancer worldwide and the sixth most common cause of cancer-related mortality [1]. Esophagectomy is the mainstay of treatment for resectable esophageal cancer. Thoracoscopic esophagectomy was first reported by Cuschieri et al. in 1992 [2]. However, thoracoscopic esophagectomy has been widely used worldwide as a curative surgery for esophageal cancer. Moreover, it reduces respiratory complications compared with open esophagectomy in a randomized controlled trial [3]. However, complications such as anastomotic leakage and stenosis remain a major concern. At our institution, thoracoscopic esophagectomy was initially performed in November 2009. Different anastomotic methods such as hand-sewing, triangulating [4], circular stapling, 
and Collard anastomosis [5] have been applied. However, the anastomotic complication rate was not reduced.

A systematic review has reported that indocyanine green (ICG) fluorescence imaging can be an important adjunct tool for reducing anastomotic leakage after esophagectomy [6]. ICG is a water-soluble nearinfrared phosphor that has immediate and long-term safety [7, 8]. ICG fluorescence imaging is a simple evaluation method. In recent robot-assisted surgery, high-resolution near-infrared images can be obtained using the Firefly system with the da Vinci Xi surgical robot (Intuitive Surgical Inc., Sunnyvale, California). Therefore, to assess gastric tube blood flow during esophagectomy reconstruction, we have standardized the use of ICG fluorescence imaging at our institution in July 2018. In addition, the anastomotic method was standardized to circular stapling anastomosis because it is simple and applicable to almost all cases, including those with short remnant esophagus.

The current study aimed to evaluate the efficacy of circular stapling anastomosis with ICG fluorescence imaging for cervical esophagogastric anastomosis after thoracoscopic esophagectomy by comparing the short-term outcomes before and after the anastomotic method was standardized via propensity matched analysis.

\section{Methods}

\section{Patients}

We included 145 patients with esophageal cancer who underwent thoracoscopic esophagectomy with radical lymph node dissection between November 2009 and December 2020 at Tottori University Hospital. Among them, 19, 2, and 3 who underwent reconstruction using the jejunum or colon, pharyngeal gastric tube anastomosis due to the simultaneous duplication of hypopharyngeal cancer, and two-stage reconstruction, respectively, were excluded. Finally, 121 patients were enrolled in the current study (Fig. 1). Patients who underwent surgery until June 2018, which was before the anastomotic method was standardized, were included in the classical group, and those who underwent surgery starting July 2018, which was after the standardization, in the ICG circular group. The clinicopathological findings were determined according to the Japanese Classification of Esophageal Cancer (11th edition) [9] [10]. This study was approved by the institutional review board (20A234), and the need for informed consent was waived.

\section{Surgical procedure}

All patients underwent thoracoscopic subtotal esophagectomy with mediastinal lymph node dissection in the prone position under right pneumothorax, and robot-assisted esophagectomy has been used since February 2020. After completion of the thoracic procedure, patients were subsequently repositioned in the supine position, and cervical and abdominal procedures were started simultaneously. Cervical lymph node dissection was not performed on patients with lower thoracic or abdominal esophageal cancer 
without cervical or upper mediastinal lymph node metastasis. Abdominal procedures such as laparotomy, hand-assisted laparoscopic surgery, and complete laparoscopy for abdominal lymph node dissection were performed. In patients who require complete laparoscopy, 8-cm laparotomy was performed on the upper abdomen after completing abdominal lymph node dissection. The gastric tube was created under direct visualization in all cases. Then, it was pulled up to the neck via the retrosternal or posterior mediastinal route, and esophagogastric anastomosis was performed on the left side of the neck.

\section{ICG circular anastomosis method}

The ICG circular anastomosis was performed as follows: After the gastric tube was created, ICG at a dose of $10 \mathrm{mg} /$ body was administered intravenously, and ICG fluorescence imaging of the blood flow in the gastric tube was assessed using PhotoDynamic Eye (Hamamatsu Photonics, Hamamatsu, Japan) or the Firefly system, which was integrated with the da Vinci Xi surgical robot (Intuitive Surgical Inc., Sunnyvale, California). The reconstructed gastric tube was used until the site where the wall of the gastric tube had a uniform contrast within $20 \mathrm{~s}$ after the right gastroepiploic artery was contrasted with ICG, as reported by Noma et al. [11] (Figs. 2a, b). After the gastric tube was pulled up to the neck, end-to-side esophagogastric anastomosis was performed on the posterior wall of the gastric tube using the 25-mm DST Series EEA circular stapler (Medtronic, Minneapolis, Minnesota) (Fig. 2c). The stump of the gastric tube was sectioned and closed using the Signia stapling system with a 60-mm purple cartridge (Medtronic, Minneapolis, Minnesota) (Fig. 2d). Finally, the staple line was buried.

\section{Definition of perioperative complications}

Anastomotic leakage was defined as saliva leakage from the cervical wound, contrast leakage outside the gastrointestinal tract on gastrointestinal series, and abnormal air or fluid accumulation around the anastomosis on CT scan. Anastomotic stenosis was defined as cases in which a 9.0-mm-diameter endoscope could not pass through the anastomosis and balloon dilation was required during endoscopy for postoperative dysphagia. Pneumonia was defined as the appearance of consolidation on chest radiography or CT scan and the detection of bacteria on sputum culture. Recurrent nerve paralysis was assessed by an otolaryngologist on postoperative day 6 or 7 via laryngoscopy.

\section{Statistical analysis}

Continuous data were presented as mean \pm standard deviation or median with range, as indicated. The Mann-Whitney $U$ test and the $\chi^{2}$ test were used to evaluate differences in continuous and categorical variables, respectively. Propensity matched analysis was conducted using the logistic regression model and covariates such as age, sex, histological type, tumor location, clinical stage, and presence or absence of neoadjuvant chemotherapy. Univariate and multivariate logistic regression analyses were used to identify risk factors for anastomotic leakage. Variables that were considered statistically significant in the univariate analysis were used in the multivariate analysis. $P$ values of $<0.05$ indicated statistically significant differences, and the Statistical Package for the Social Sciences software version 25 (IBM SPSS Inc., Chicago, Illinois) was used for statistical analyses. 


\section{Results}

\section{Characteristics of patients}

Of 121 patients, 82 were included in the classical group and 39 in the ICG circular group before matching. Then, 33 patients were respectively included in each group (Fig. 1). Table 1 shows the clinicopathological characteristics of patients before and after matching. Before matching, there were significant differences in terms of the American Society of Anesthesiologists physical status (ASA-PS) score $(P=0.021)$ and histological type $(P=0.009)$. However, after matching, the background characteristics did not significantly differ between the two groups. 
Table 1

Characteristics of patients

Before matching

\begin{tabular}{llllll}
$\begin{array}{l}\text { Classical } \\
\text { group }\end{array}$ & $\begin{array}{l}\text { ICG circular } \\
\text { group }\end{array}$ & $\begin{array}{l}P \\
\text { value }\end{array}$ & $\begin{array}{l}\text { Classical } \\
\text { group }\end{array}$ & $\begin{array}{l}\text { ICG circular } \\
\text { group }\end{array}$ & $\begin{array}{l}P \\
\text { value }\end{array}$ \\
\hline$(n=82)$ & $(n=39)$ & & $(n=33)$ & $(n=33)$ & \\
\hline
\end{tabular}

Age (years)

Median (range)

$66(49-\quad 66(41-79)$

81)

Sex

Male

Female

Body mass index

$\left(\mathrm{kg} / \mathrm{m}^{2}\right)$

Serum albumin leve $(\mathrm{g} / \mathrm{dL})$

Brinkman index

Median (range)

Performance status

0

1

2

Comorbidity

Diabetes

Cardiovascular

disease

Obstructive

ventilation failure

ASA-PS score

1

2
$15(18 \%) \quad 4(10 \%)$

$10(12 \%) \quad 6(15 \%) \quad 0.628$

$27(33 \%) \quad 13(33 \%)$

0.965

0.021

\begin{tabular}{|c|c|c|c|c|}
\hline ASA-PS score & & & & \\
\hline 1 & $11(13 \%)$ & $1(3 \%)$ & 3 (9\%) & $1(3 \%)$ \\
\hline 2 & $63(77 \%)$ & $28(72 \%)$ & 27 (82\%) & $23(70 \%)$ \\
\hline
\end{tabular}

After matching

0.872

$65(49-\quad 67(41-79)$

79)

0.282

30 (90\%) 27 (82\%)

$3(9 \%) \quad 6(18 \%)$

$21.8 \pm 3.1 \quad 21.9 \pm 3.3 \quad 0.677$

$4.1 \pm 0.4 \quad 4.1 \pm 0.4$

0.985

0.733

0.278

$820(0-$ 2600)

$840(0-$ 1250)

0.586

0.601

$27(82 \%) \quad 28(85 \%)$

$5(15 \%) \quad 5(15 \%)$

$1(3 \%) \quad 0(0 \%)$

ASA-PS, American Society of Anesthesiologists physical status 


\begin{tabular}{|c|c|c|c|c|c|c|}
\hline \multirow[b]{2}{*}{3} & \multicolumn{3}{|c|}{ Before matching } & \multicolumn{3}{|c|}{ After matching } \\
\hline & $8(10 \%)$ & $10(26 \%)$ & & $3(9 \%)$ & $9(27 \%)$ & \\
\hline Histological type & & & 0.009 & & & 1.000 \\
\hline $\begin{array}{l}\text { Squamous cell } \\
\text { carcinoma }\end{array}$ & 78 (95\%) & $30(77 \%)$ & & 30 (91\%) & 30 (91\%) & \\
\hline Adenocarcinoma & $2(2 \%)$ & $6(15 \%)$ & & $2(6 \%)$ & $2(6 \%)$ & \\
\hline Others & $2(2 \%)$ & $3(8 \%)$ & & $1(3 \%)$ & $1(3 \%)$ & \\
\hline Tumor location & & & 0.229 & & & 0.642 \\
\hline Upper thoracic & $11(13 \%)$ & $6(15 \%)$ & & $5(15 \%)$ & $6(18 \%)$ & \\
\hline Middle thoracic & $43(52 \%)$ & $16(41 \%)$ & & $15(46 \%)$ & $16(49 \%)$ & \\
\hline Lower thoracic & $24(29 \%)$ & $11(28 \%)$ & & $11(33 \%)$ & $7(21 \%)$ & \\
\hline Abdominal & $4(5 \%)$ & $6(15 \%)$ & & $2(6 \%)$ & $4(12 \%)$ & \\
\hline cT & & & 0.405 & & & 0.667 \\
\hline 1 & $36(44 \%)$ & $16(41 \%)$ & & $16(49 \%)$ & $14(42 \%)$ & \\
\hline 2 & $14(17 \%)$ & $11(28 \%)$ & & $5(15 \%)$ & $9(27 \%)$ & \\
\hline 3 & 31 (38\%) & $11(28 \%)$ & & $11(33 \%)$ & $9(27 \%)$ & \\
\hline $4 a$ & $1(1 \%)$ & $1(3 \%)$ & & $1(3 \%)$ & $1(3 \%)$ & \\
\hline $\mathrm{cN}$ & & & 0.224 & & & 0.420 \\
\hline 0 & $44(54 \%)$ & $28(72 \%)$ & & 19 (58\%) & 24 (73\%) & \\
\hline 1 & $17(21 \%)$ & $5(13 \%)$ & & $7(21 \%)$ & $4(12 \%)$ & \\
\hline 2 & $20(24 \%)$ & $5(13 \%)$ & & $7(21 \%)$ & $5(15 \%)$ & \\
\hline 3 & $1(1 \%)$ & $1(3 \%)$ & & $0(0 \%)$ & $0(0 \%)$ & \\
\hline cStage & & & 0.296 & & & 0.393 \\
\hline 1 & 32 (39\%) & $14(36 \%)$ & & $16(49 \%)$ & 13 (39\%) & \\
\hline 2 & 19 (23\%) & $14(36 \%)$ & & $5(15 \%)$ & $10(30 \%)$ & \\
\hline 3 & 31 (38\%) & $11(28 \%)$ & & $12(36 \%)$ & $10(30 \%)$ & \\
\hline $\begin{array}{l}\text { Neoadjuvant } \\
\text { chemotherapy }\end{array}$ & & & 0.974 & & & 1.000 \\
\hline Absent & 36 (44\%) & 17 (44\%) & & 16 (49\%) & 16 (49\%) & \\
\hline
\end{tabular}




\begin{tabular}{|c|c|c|c|c|}
\hline \multirow[b]{2}{*}{ Present } & \multicolumn{2}{|c|}{ Before matching } & \multicolumn{2}{|c|}{ After matching } \\
\hline & $46(56 \%)$ & $22(56 \%)$ & $17(52 \%)$ & 17 (52\%) \\
\hline
\end{tabular}

\section{Changes in anastomotic methods and perioperative outcomes}

Figure 3 presents changes in anastomotic methods for cervical esophagogastric anastomosis after thoracoscopic esophagectomy, and Table 2 depicts the perioperative outcomes in both groups. As shown in Table 2, before matching, there was a significantly higher proportion of patients who underwent surgery using the laparoscopic approach $(P<0.001)$ and narrow gastric tube $(P=0.001)$ and those who had a lower volume of blood loss $(P=0.038)$ in the ICG circular group. After matching, the same factor indicated a significant difference. In terms of postoperative outcomes, the ICG circular group had a significantly lower proportion of patients with anastomotic leakage ( $34 \% \mathrm{vs.} 8 \%, P=0.002)$ and shorter postoperative hospital stay ( 29 vs. 20 days, $P<0.001)$ before matching. After matching, there was a significantly lower proportion of patients with anastomotic leakage ( $39 \%$ vs. $9 \%, P=0.004)$ and stenosis ( $46 \%$ vs. $21 \%, P=0.037$ ) and a shorter postoperative hospital stay (30 vs. 20 days, $P<0.001)$ in the ICG circular group. 
Table 2

Perioperative outcomes of patients with esophageal cancer after thoracoscopic esophagectomy

\section{Before matching}

\begin{tabular}{llllll}
$\begin{array}{l}\text { Classical } \\
\text { group }\end{array}$ & $\begin{array}{l}\text { ICG circular } \\
\text { group }\end{array}$ & $\begin{array}{l}P \\
\text { value }\end{array}$ & $\begin{array}{l}\text { Classical } \\
\text { group }\end{array}$ & $\begin{array}{l}\text { ICG } \\
\text { circular } \\
\text { group }\end{array}$ & $\begin{array}{l}P \\
\text { value }\end{array}$ \\
\hline$(n=82)$ & $(n=39)$ & & $(n=33)$ & $(n=33)$ &
\end{tabular}

Abdominal

approach

Open

Laparoscopic

Lymph node

dissection

Two-field

Three-field

Route of

reconstruction

Retrosternal

Posterior

mediastinal

Shape of the gastric

tube

Subtotal

Narrow

Total operative time $\quad 634 \pm 89$

(min)

Volume of blood

loss $(\mathrm{mL})$

Postoperative

complications

Anastomotic

leakage

Anastomotic

stenosis

Pneumonia

$18(22 \%) \quad 13(33 \%)$

$<0.001$

$34(42 \%) \quad 0(0 \%)$

$48(59 \%) \quad 39(100 \%)$

$64(78 \%) \quad 26(67 \%)$

$10(30 \%) \quad 10(30 \%)$

$23(70 \%) \quad 23(70 \%)$

0.070

$15(46 \%) \quad 0(0 \%)$

$18(55 \%) \quad 33(100 \%)$

0.180

1.000

0.001

0.131

68 (83\%) $\quad 37$ (95\%)

$27(82 \%) \quad 31(94 \%)$

$6(18 \%) \quad 2(6 \%)$

$14(17 \%) \quad 2(5 \%)$

0.003

0.001

$0(0 \%)$

$21(26 \%) \quad 0(0 \%)$

$8(24 \%)$

$33(100 \%)$

$61(74 \%) \quad 39(100 \%)$

$25(76 \%)$

$616 \pm 51$

0.753

$617 \pm 53$

0.573

$638 \pm$

100

$186 \pm 219 \quad 103 \pm 97$

0.038

$251 \pm$

$93 \pm 89$

0.009

298

$28(34 \%) \quad 3(8 \%)$

0.002

$13(39 \%) \quad 3(9 \%)$

0.004

$29(35 \%) \quad 8(21 \%)$

0.097

$15(46 \%) \quad 7(21 \%)$

0.037

$18(22 \%) \quad 11(28 \%)$

0.451

$8(24 \%)$

$9(27 \%)$

0.778 


\begin{tabular}{|lllllll|}
\hline \multicolumn{4}{l}{ Before matching } & \multicolumn{4}{c|}{ After matching } \\
\hline $\begin{array}{l}\text { Recurrent nerve } \\
\text { paralysis }\end{array}$ & $14(17 \%)$ & $2(5 \%)$ & 0.070 & $7(21 \%)$ & $2(6 \%)$ & 0.073 \\
\hline $\begin{array}{l}\text { Postoperative } \\
\text { hospital stay }\end{array}$ & $29(10-$ & $20(14-53)$ & $<0.001$ & $\begin{array}{l}30(10- \\
128)\end{array}$ & $20(14-53)$ & $<$ \\
\hline
\end{tabular}

\section{Risk factor analyses of anastomotic leakage}

Finally, we evaluated the risk factors for anastomotic leakage via propensity score matching in 66 patients. The univariate analysis indicated that Brinkman index $(P=0.048)$ and anastomotic method $(P=$ 0.008 ) were significantly associated with anastomotic leakage (Table 3 ). In the multivariate analysis, anastomotic method was an independent risk factor for anastomotic leakage after thoracoscopic esophagectomy (odds ratio: 5.983, 95\% confidence interval [Cl]: 1.469-24.359, $P=0.013$ ) (Table 4). 
Table 3

Univariate logistic regression analyses of anastomotic leakage

\section{Anastomotic leakage}

$\begin{array}{llll}\text { Absent }(n= & \begin{array}{l}\text { Present }(n= \\ \text { 50) }\end{array}(n)\end{array} \quad$ OR $\quad 95 \% \mathrm{Cl} \quad \begin{aligned} & P \\ & \text { value }\end{aligned}$

Age (years)

$\begin{array}{llllll}<65 & 20(40 \%) & 8(50 \%) & 1.500 & 0.484-4.651 & 0.483 \\ \geq 65 & 30(60 \%) & 8(50 \%) & 1 & & \\ \text { Sex } & & & & & \\ \text { Male } & 42(84 \%) & 15(94 \%) & 2.857 & 0.329- & 0.341 \\ \text { Female } & 8(16 \%) & 1(6 \%) & 1 & & \end{array}$

Body mass index $\left(\mathrm{kg} / \mathrm{m}^{2}\right)$

$\begin{array}{llllll}<22 & 24(48 \%) & 10(63 \%) & 1.806 & 0.569-5.726 & 0.316 \\ \geq 22 & 26(52 \%) & 6(38 \%) & 1 & \end{array}$

Serum albumin level $(\mathrm{g} / \mathrm{dL})$

$\begin{array}{llllll}<4 & 20(40 \%) & 5(31 \%) & 0.682 & 0.206-2.261 & 0.531 \\ \geq 4 & 30(60 \%) & 11(69 \%) & 1 & \end{array}$

Brinkman index

$\begin{array}{llllll}<800 & 24(48 \%) & 3(19 \%) & 0.250 & 0.063-0.986 & 0.048 \\ \geq 800 & 26(52 \%) & 13(81 \%) & 1 & \end{array}$

Performance status

$\begin{array}{llllll}0 & 42(84 \%) & 13(81 \%) & 0.825 & 0.191-3.574 & 0.797 \\ 1,2 & 8(16 \%) & 3(19 \%) & 1 & & \end{array}$

Diabetes

$\begin{array}{lccccc}\text { Absent } & 39(78 \%) & 13(81 \%) & 1.222 & 0.295-5.069 & 0.782 \\ \text { Present } & 11(22 \%) & 3(19 \%) & 1 & & \\ \text { Cardiovascular disease } & & & & & \\ \text { Absent } & 46(92 \%) & 13(81 \%) & 0.377 & 0.075-1.901 & 0.237\end{array}$

OR, odds ratio; $\mathrm{Cl}$, confidence interval; ASA-PS, American Society of Anesthesiologists physical status 


\section{Anastomotic leakage}

Present

Obstructive ventilation

failure

$\begin{array}{llllll}\text { Absent } & 36(72 \%) & 9(56 \%) & 0.500 & 0.156-1.603 & 0.243 \\ \text { Present } & 14(28 \%) & 7(44 \%) & 1 & & \end{array}$

ASA-PS score

1

2,3

Histological type

Squamous cell carcinoma

Others

Tumor location

$\mathrm{Ut}, \mathrm{Mt}$

Lt, Ae

CT

1

$2,3,4 a$

$\mathrm{cN}$

$\begin{array}{llllll}\text { Absent } & 33(66 \%) & 10(63 \%) & 0.859 & 0.267-2.764 & 0.798 \\ \text { Present } & 17(34 \%) & 6(38 \%) & 1 & & \end{array}$

cStage

1

2,3

Neoadjuvant chemotherapy

Absent

Present

OR, odds ratio; $\mathrm{Cl}$, confidence interval; ASA-PS, American Society of Anesthesiologists physical status 


\section{Anastomotic leakage}

Abdominal approach

\begin{tabular}{|c|c|c|c|c|c|}
\hline Open & $11(22 \%)$ & $4(25 \%)$ & 1.182 & $0.317-4.400$ & 0.803 \\
\hline Laparoscopic & $39(78 \%)$ & $12(75 \%)$ & 1 & & \\
\hline \multicolumn{6}{|l|}{ Lymph node dissection } \\
\hline Two-field & $15(30 \%)$ & $5(31 \%)$ & 1.061 & $0.314-3.585$ & 0.925 \\
\hline Three-field & $35(70 \%)$ & $11(69 \%)$ & 1 & & \\
\hline \multicolumn{6}{|l|}{ Route of reconstruction } \\
\hline Retrosternal & $43(86 \%)$ & $15(94 \%)$ & 2.442 & $\begin{array}{l}0.277- \\
21.519\end{array}$ & 0.421 \\
\hline Posterior mediastinal & $7(14 \%)$ & $1(6 \%)$ & 1 & & \\
\hline \multicolumn{6}{|l|}{ Shape of gastric tube } \\
\hline Subtotal & $5(10 \%)$ & $3(19 \%)$ & 2.077 & $0.437-9.871$ & 0.358 \\
\hline Narrow & $45(90 \%)$ & $13(81 \%)$ & 1 & & \\
\hline \multicolumn{6}{|c|}{ Total operative time (min) } \\
\hline$<600$ & $20(40 \%)$ & $6(38 \%)$ & 0.900 & $0.282-2.870$ & 0.859 \\
\hline$\geq 600$ & $30(60 \%)$ & $10(63 \%)$ & 1 & & \\
\hline \multicolumn{6}{|l|}{ Blood loss (mL) } \\
\hline$<100$ & $26(52 \%)$ & $7(44 \%)$ & 0.718 & $0.231-2.229$ & 0.566 \\
\hline$\geq 100$ & $24(48 \%)$ & $9(56 \%)$ & 1 & & \\
\hline \multicolumn{6}{|l|}{ Anastomotic method } \\
\hline ICG circular group & $30(60 \%)$ & $3(19 \%)$ & 0.154 & $0.039-0.610$ & 0.008 \\
\hline Classical group & $20(40 \%)$ & $13(81 \%)$ & 1 & & \\
\hline
\end{tabular}

OR, odds ratio; $\mathrm{Cl}$, confidence interval; ASA-PS, American Society of Anesthesiologists physical status 
Table 4

Multivariate logistic regression analyses of anastomotic leakage

\begin{tabular}{|llll|}
\hline & OR & $95 \% \mathrm{Cl}$ & Pvalue \\
\hline Brinkman index $(\geq 800)$ & 3.538 & $0.842-14.860$ & 0.084 \\
\hline Anastomotic method (classical group) & 5.983 & $1.469-24.359$ & 0.013 \\
\hline OR, odds ratio; Cl, confidence interval & & & \\
\hline
\end{tabular}

\section{Discussion}

The current study aimed to evaluate the efficacy of circular stapling anastomosis with ICG fluorescence imaging for cervical esophagogastric anastomosis after thoracoscopic esophagectomy by comparing the short-term outcomes before and after the anastomotic method was standardized via propensity matched analysis. The ICG circular group had a significantly lower rate of complications, including anastomotic leakage and stenosis, and a shorter postoperative hospital stay. Furthermore, anastomotic method was an independent risk factor for anastomotic leakage after thoracoscopic esophagectomy.

The incidence of anastomotic leakage was significantly lower in the ICG circular group than in the classical group, and anastomotic method was an independent risk factor for postoperative anastomotic leakage. According to a systematic review of ICG fluorescence imaging after esophageal cancer surgery, the overall anastomotic leakage rate of patients who underwent surgery with ICG fluorescence imaging was lower than that of controls (13.5\% [118/873] vs. 18.5\% [86/466]) [6]. Furthermore, when anastomosis was performed on the area with good ICG perfusion, the incidence of anastomotic leakage was $9.0 \%$ (67/746). In our current study, anastomosis was performed on the area of good ICG perfusion in all patients in the ICG circular group. Thus, the outcomes of anastomotic leakage were comparable. Honda et al. performed a systematic review comparing anastomosis after esophagectomy between hand-sewn and mechanical anastomosis with a circular stapler [12]. Results showed that the anastomotic leakage rate with circular stapling anastomosis $(6.1 \%, 41 / 668)$ was similar to that with hand-sewn anastomosis $(6.1 \%, 39 / 640)$ (risk ratio [RR]: $1.02,95 \% \mathrm{Cl}: 0.66-1.59, P=0.43)$. Moreover, both anastomotic methods had a significantly lower rate. However, this review included studies performing intrathoracic anastomosis. Hence, the outcomes were not completely comparable to those of cervical esophagogastric anastomosis, and our results are considered acceptable. In addition, Honda et al. showed that mechanical circular stapling anastomosis significantly reduced the operative time by 15.3 min compared with hand-sewn anastomosis. In our study, total operative time tended to be shorter in the ICG circular group than in the classical group; however, it was not significant. Therefore, circular stapling anastomosis with ICG fluorescence imaging is a simple and safe method that can reduce anastomotic leakage after thoracoscopic esophagectomy.

The current study showed that the incidence of anastomotic stenosis was significantly lower in the ICG circular group than in the classical group after propensity score matching. However, the anastomotic 
stenosis rate in the classical group after matching was extremely high at $46 \%$, which might had a significant impact. Honda et al. showed that the stenosis rate of circular stapling anastomosis was significantly higher than that of hand-sewn anastomosis (16.9\% [106/626] and 9.9\% [62/629]) (RR: 1.67, $95 \% \mathrm{Cl}: 1.16-2.42, P=0.006)$. The subgroup and meta-regression analysis showed no significant differences in the anastomotic site, diameter of the circular stapler, layer, and configuration [12]. A randomized controlled trial by Hayata et al. comparing cervical esophagogastric circular stapling and triangulating stapling anastomosis after esophagectomy showed no significant difference in terms of anastomotic stenosis rate between the circular stapling group $(17 \%, 8 / 47)$ and the triangulating stapling group $(19 \%, 9 / 51)(P=0.935)[13]$. In the current study, the stenosis rate of circular stapling anastomosis was $21 \%$, which is similar to that of previous reports but not satisfactory. Therefore, although the incidence rate of anastomotic stenosis is improving due to the standardization of anastomotic methods, it should still be further reduced.

The current study had several limitations. First, this was a retrospective study with a small sample size. Reconstruction-related perioperative outcomes, such as abdominal approach and shape of gastric tube were not consistent between the two groups. Therefore, the outcomes of ICG circular anastomosis after standardization must be prospectively evaluated. Second, various anastomotic methods were included in the classical group. Therefore, propensity matched analysis was performed to eliminate differences in patient characteristics and to prevent bias as much as possible.

\section{Conclusions}

Circular stapling anastomosis with ICG fluorescence imaging is effective in reducing anastomotic complications for cervical esophagogastric anastomosis after thoracoscopic esophagectomy. However, the incidence rate of anastomotic stenosis can still be improved, and this is an issue that should be addressed in the future.

\section{List Of Abbriviations}

ICG: indocyanine green

ASA-PS: American Society of Anesthesiologists physical status

Cl: confidence interval

RR: risk ratio

\section{Declarations}

\section{Ethics approval and consent to participate:}


This study was approved by the ethical review board, Tottori University School of Medicine (20A234), waived the informed consent, and performed in accordance with the Declaration of Helsinki.

\section{Consent for publication:}

Not applicable.

\section{Availability of data and materials:}

The datasets generated during and analyzed during the current study are not publicly available due to difficulty in obtaining approval from all patients for the release of detailed personal data, but are available from the corresponding author on reasonable request.

\section{Competing interests:}

The authors declare that they have no competing interests.

\section{Funding:}

The authors received no funding for this study.

\section{Authors' contributions:}

Study conception: YS; Study design: YS and TM; Data acquisition: YS, TM, MM, WM, SS and KM; Quality control of data and algorithms: CU, MM, YM, TH and KK; Data analysis and interpretation: MY, NT, ST and TS; Statistical analysis: YS; Manuscript preparation: YS; Manuscript editing: HS and TH; Manuscript review: TM and YF; Final approval of the article: all authors.

\section{Acknowledgements:}

The authors would like to thank Enago (www.enago.jp) for the English language review.

\section{References}

1. Bray F, Ferlay J, Soerjomataram I, Siegel RL, Torre LA, Jemal A. Global cancer statistics 2018 : GLOBOCAN estimates of incidence and mortality worldwide for 36 cancers in 185 countries. CA Cancer J Clin. 2018;68:394-424. doi: 10.3322/caac.21492. 
2. Cuschieri A, Shimi S, Banting S. Endoscopic oesophagectomy through a right thoracoscopic approach. J R Coll Surg Edinb. 1992;37:7-11.

3. Biere SS, van Berge Henegouwen MI, Maas KW, Bonavina L, Rosman C, Garcia JR, et al. Minimally invasive versus open oesophagectomy for patients with oesophageal cancer: a multicentre, openlabel, randomised controlled trial. Lancet. 2012;379:1887-92. doi: 10.1016/S0140-6736(12)60516-9.

4. Toh Y, Sakaguchi Y, Ikeda O, Adachi E, Ohgaki K, Yamashita Y, et al. The triangulating stapling technique for cervical esophagogastric anastomosis after esophagectomy. Surg Today. 2009;39:201-6. doi: 10.1007/s00595-008-3827-2.

5. Collard JM, Romagnoli R, Goncette L, Otte JB, Kestens PJ. Terminalized semimechanical side-to-side suture technique for cervical esophagogastrostomy. Ann Thorac Surg. 1998;65(3):814-7. doi: 10.1016/s0003-4975(97)01384-2.

6. Koyanagi K, Ozawa S, Ninomiya Y, Yatabe K, Higuchi T, Yamamoto M, et al. Indocyanine green fluorescence imaging for evaluating blood flow in the reconstructed conduit after esophageal cancer surgery. Surg Today. 2021. doi: 10.1007/s00595-021-02296-4.

7. Ogawa $\mathrm{M}$, Kosaka $\mathrm{N}$, Choyke $\mathrm{PL}$, Kobayashi $\mathrm{H}$. In vivo molecular imaging of cancer with a quenching near-infrared fluorescent probe using conjugates of monoclonal antibodies and indocyanine green. Cancer Res. 2009;69:1268-72. doi: 10.1158/0008-5472.CAN-08-3116.

8. Murawa D, Polom K, Murawa P. One-year postoperative morbidity associated with near-infraredguided indocyanine green (ICG) or ICG in conjugation with human serum albumin (ICG:HSA) sentinel lymph node biopsy. Surg Innov. 2014;21:240-3. doi: 10.1177/1553350613503737.

9. Japanese Classification of Esophageal Cancer. 11th ed. (I). Esophagus: official journal of the Japan Esophageal Society. 2017;14:1-36.

10. Japanese Classification of Esophageal Cancer. 11th ed. (II and III). Esophagus: official journal of the Japan Esophageal Society. 2017;14:37-65.

11. Noma K, Shirakawa Y, Kanaya N, Okada T, Maeda N, Ninomiya T, et al. Visualized evaluation of blood flow to the gastric conduit and complications in esophageal reconstruction. J Am Coll Surg. 2018;226:241-51. doi: 10.1016/j.jamcollsurg.2017.11.007.

12. Honda M, Kuriyama A, Noma $H$, Nunobe $S$, Furukawa TA. Hand-sewn versus mechanical esophagogastric anastomosis after esophagectomy: a systematic review and meta-analysis. Ann Surg. 2013;257:238-48. doi: 10.1097/SLA.0b013e31826d4723.

13. Hayata K, Nakamori M, Nakamura M, Ojima T, Iwahashi M, Katsuda M, et al. Circular stapling versus triangulating stapling for the cervical esophagogastric anastomosis after esophagectomy in patients with thoracic esophageal cancer: A prospective, randomized, controlled trial. Surgery. 2017;162:1318. doi: 10.1016/j.surg.2017.01.013.

\section{Figures}




\section{Figure 1}

Patient selection for the evaluation of cervical esophagogastric anastomosis after thoracoscopic esophagectomy

\section{Figure 2}

Procedures in ICG circular anastomosis

(a) The right gastroepiploic artery was contrasted with ICG.

(b) The site where the wall of the gastric tube had a uniform contrast with ICG.

(c) End-to-side esophagogastric anastomosis was performed on the posterior wall of the gastric tube using the 25-mm DST Series EEA circular stapler.

(d) The stump of the gastric tube was sectioned and closed using the Signia stapling system with a 60mm purple cartridge.

\section{Figure 3}

Changes in anastomotic methods for cervical esophagogastric anastomosis after thoracoscopic esophagectomy

Changes in the classical and ICG circular groups, and the annual incidence rates of anastomotic leakage and stenosis in anastomotic methods for cervical esophagogastric anastomosis after thoracoscopic esophagectomy are shown. 